\title{
Lung Ultrasound in Hemodialysis: A Card to be Played?
}

\author{
Pierpaolo Di Nicolò ${ }^{a}$ Giacomo Magnoni ${ }^{b}$ Antonio Granata ${ }^{c}$ \\ aNephrology and Dialysis Unit, "S. Maria della Scaletta" Hospital, Imola, ${ }^{b}$ Nephrology and Dialysis Unit, \\ "Sondrio Civil Hospital," Sondrio, and "Nephrology and Dialysis Unit, "S. Giovanni di Dio" Hospital, Agrigento, Italy
}

\section{Keywords}

Hemodialysis · Dry weight · Lung interstitial syndrome · Lung ultrasound

\begin{abstract}
The end-stage renal disease is characterized by a profound impairment in the regulation of body fluid distribution, and volume assessment in hemodialysis is one of the challenging goals for the nephrologist. To determine a state of euvolemia, different validated techniques have been employed and among them lung ultrasonography (LUS) has recently attracted growing attention on account of its capacity to estimate accurately extra vascular lung water and to detect lung edema even in its early asymptomatic stage, that is, hidden lung congestion. With its noninvasiveness, freedom from radiation, the ease of use, acceptable intra/inter-operator reproducibility and availability of portable ultrasound devices, LUS can be considered one of the most interesting "cards to play" for the volume assessment in patients on hemodialysis.

๑) 2017 S. Karger AG, Basel
\end{abstract}

\section{Introduction}

The end-stage renal disease (ESRD) is characterized by a profound impairment in the regulatory system of body fluid with important aftermath in terms of survival for the risk of acute and chronic volume overload [1].

\section{KARGER}

(c) 2017 S. Karger AG, Basel

E-Mail karger@karger.com

www.karger.com/bpu
As a consequence, volume assessment in hemodialysis is one of the primary goals, often challenging, that is to be achieved by the nephrologist.

To determine the euvolemic status together with clinical evaluation, often inaccurate and subjective, various and novel techniques are employed, sometimes together, in a comprehensive assessment strategy previously proposed as the " $5 \mathrm{~B}$ " approach: body weight changes, blood volume online monitoring, bioimpedance spectroscopy (BIS), B-natriuretic peptide and body ultrasound (US), which includes inferior vena cava (IVC) diameter measure and lung US (LUS) [2]. Among them, LUS has recently attracted growing attention, thanks to its capacity to estimate accurately the lung congestion in ESRD by measuring extravascular lung water (EVLW).

Thanks to its noninvasiveness, free from radiation, the ease of use, acceptable intra/inter-operator reproducibility and to the availability of portable US devices, chest ultrasonography can be considered one of the most interesting techniques offered to the nephrologist for volume assessment in ESRD [3]. But as any other clinical instrument, it has strengths and limitations that have to be recognized by the nephrologist who wants to enrich his clinical experience with this powerful tool.

The purpose of our mini-review is to clarify the physiopathological basis of LUS and its potential use in the hemodialysis setting.

Dr. Pierpaolo Di Nicolò, MD

Nephrology and Dialysis Unit, "S. Maria della Scaletta" Hospita

Via Montericco, 4

IT-40026 Imola (BO) (Italy)

E-Mail p.dinicolo@ hotmail.it 
Fig. 1. Examples of lung ultrasonography imaging. The probe is placed in the intercostal space parallel to the ribs. a Normal appearance of lung surface with a clearly distinguishable pleural line: $\mathrm{B}$-lines are $\mathrm{ab}$ sent. b The presence of 2 B-lines can be appreciated as 2 vertical reverberation artifacts originating from the pleural line. The possible detection of up to $2 \mathrm{~B}$-lines per single intercostal space may be physiological. c Several lung comets are showed here. Multiple B-lines are considered the sonographic hallmark of lung interstitial syndrome. They configure the so called " $\mathrm{B}$ pattern."

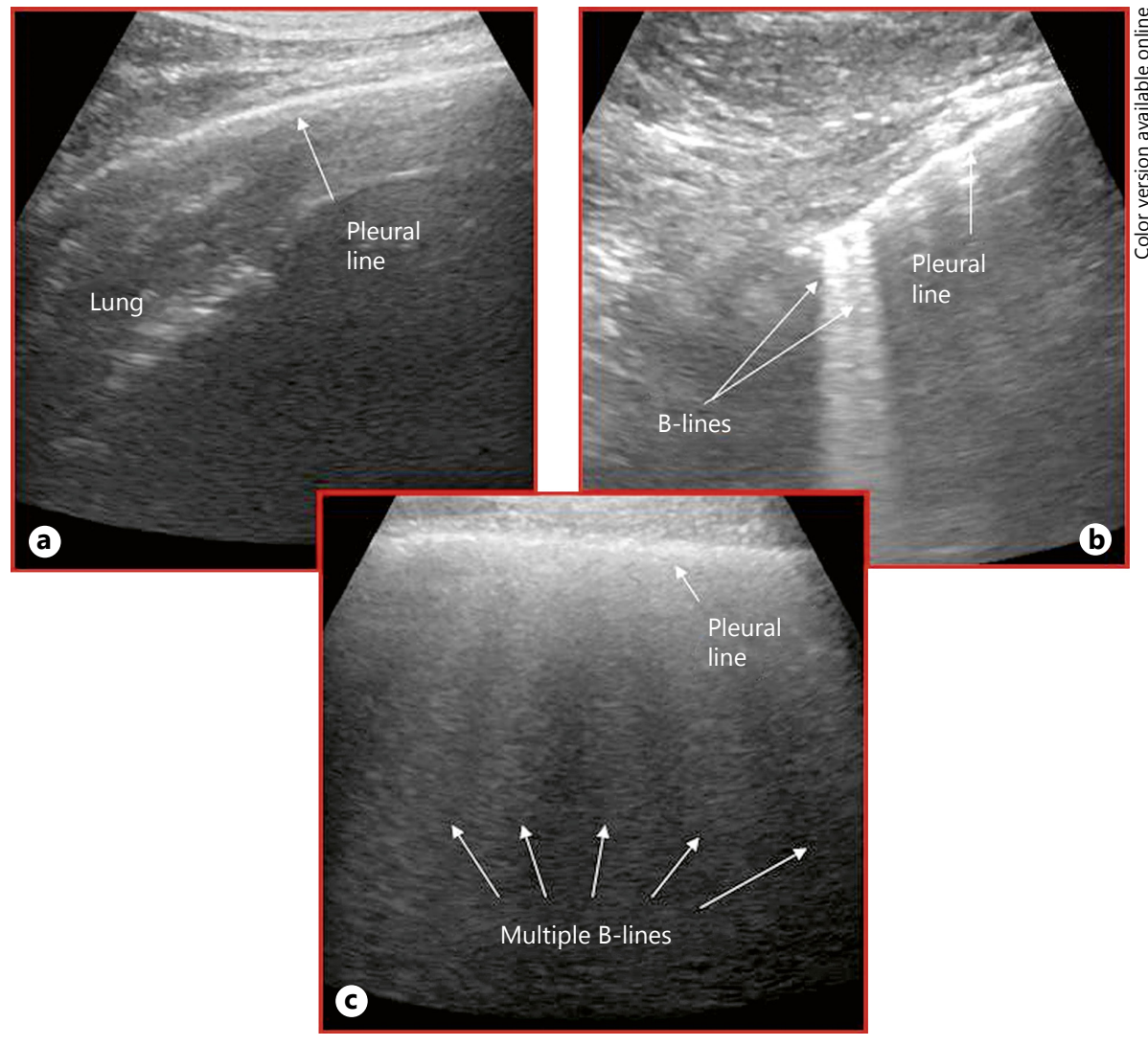

\section{B-Lines: History and Clinical Significance}

The US techniques involve the passage of US emitted by the probe through a series of physical interfaces (solid or liquid).

The presence of air prevents the transmission of the US waves and for that reason, until in recent times, the lungs were considered organs poorly or completely inexplorable with US techniques (Fig. 1a).

Nevertheless, all the pathological conditions that reduce the amount of air and conversely increase the fluid component in the pulmonary parenchyma make it amenable to examination by US.

In this case, however, the echography is not performed through the analysis of the morphology of the organ but rather through the study of "artifacts" that the disease process has generated.

Thus, we can roughly recognize 2 types of reverberation artifacts determined by the lung surface: the ring down and the comet tail artifacts.

While the first called "A-lines" are horizontal repetition artifacts without a clinical meaning, the latter, called simply "comets" or "B-lines" have a vertical trend and are of particular importance in situations of lung water overload (Fig. 1b, c).

With chest US, the typical sonographic appearance of $\mathrm{B}$-lines is of laser-like vertical hyper echoic reverberations originating from the pleural line and moving in harmony with pleural sliding [4].

The very first description of the comet tail artifacts derives from the US abdominal examination of a patient who had sustained an abdominal shotgun injury: the presence of lead pellets with their different degree of acoustic impedance among surrounding tissues determined the formation of numerous reverberation echoes [5].

The clinical application of the "comets" in the chest study appeared with the work of Lichtenstein who, having found a strong and almost exclusive association between B-lines and clinical pictures of pulmonary edema, proposed its validity as a tool for the differential diagnosis of cardiogenic dyspnea compared to that related to exacerbations of noninflammatory chronic obstructive pulmonary disease [6].

Finally, with the demonstration of a linear correlation between radiological and ultrasonographic score of lung 
overload, the B-lines became the equivalent of radiologic Kerley B-lines [7].

The EVLW, that is, the water present at the level of pulmonary interstitium is the key element for LUS and is what LUS can directly estimate.

EVLW can be strictly correlated to left ventricular (LV) filling pressures and with the escalation of the degree of pulmonary edema, the number of B-lines rises proportionally starting from the base of the thorax and rising up to the apex $[8,9]$.

However, the relationship between B-lines and capillary pressures is not exclusive and it is possible to obtain increasing comets even when pulmonary pressures are normal or near-normal such as in the cases of acute respiratory distress syndrome (ARDS) where the pivotal role for increased EVLW is played by the augmented pulmonary vascular permeability.

Curiously, though the clinical significance of lung comets is reasonably well documented, their exact anatomic and physical basis remains uncertain: the previously accepted theory is that, derivation of B-lines is from sub-pleural interlobular septa thickened by the increase in EVLW. This interpretation is currently considered too simplistic, if not erroneous $[10,11]$.

In fact, the anatomical distance between the interlobular septa is in the order of $10-30 \mathrm{~mm}$ and not $7 \mathrm{~mm}$ as it is among the comets; moreover, the comet artifacts are also observed in the absence of an identifiable object such as those that occur, for example, as in insonation of a foam object [9].

As a consequence, it is now accepted by many authors that B-lines do not have a specific anatomical basis and that they rather represent random internal reverberations caused by the interplay of interfaces among fluid-filled alveoli or, in other words, the comets would be the result of the change of water-to-air ratio per unit of insonated lung tissue determined by EVLW expansion [4, 9-11]. This explanation albeit remains somewhat inadequate.

\section{LUS Technical Aspects and Issues}

Chest US can be performed with linear, cardiac, or convex probes (with a range from 2.5 up to $7.5 \mathrm{MHz}$ ) and with the use of harmonic or fundamental imaging without important differences in terms of comets count [4, 7]. The search for the B-lines requires the exploration of various thoracic regions, ideally 4 to hemithorax or the carrying out of a total of 28 scans in the intercostal spaces (28 rib interspaces technique) $[12,13]$.

LUS in Hemodialysis
Accordingly to the latter, in order to analyze anterior and lateral parts of the chest, US explorations of both hemi-thoraxes are performed by scanning from the second to the fifth intercostal space (on the left side to the fourth intercostal space) and from the parasternal line to the axillary line for a total of 28 positions for a complete examination [14] (Fig. 2).

Using this technique, it is possible to obtain a quantitative or semi-quantitative evaluation of the interstitial lung water, thanks to the count of B-lines: in each intercostal space, comets are counted $(0-10)$ and in the case of their confluence, the percentage of the rib space covered by the B-lines and divided by 10 is estimated [4].

The interstitial syndrome is defined as the presence of bilateral multiple and diffuse B-lines. The component of EVLW is considered absent or mild when up to a total number of 15 comets are detected, moderate from 16 to 30 comets, and serious if B-lines observed exceed 30 [7].

Accordingly, some operating limits of the LUS technique have to be considered.

Chest US may be hindered by the presence of large dressings, by possible subcutaneous emphysema and by soft tissue swelling or obesity and objective quantitation of the B-lines can be difficult $[10,15]$.

Furthermore, the thoracic US does not combine its high sensitivity to an equally satisfying specificity. Therefore, the same sonographic appearance of numerous and scattered comets, called "B pattern," can be caused by EVLW expansion of varying origin, from a diffuse interstitial pneumonia to ARDS $[4,16]$.

Moreover, diffuse parenchymal lung disease associated with thickening of sub-pleural structures, such as pulmonary fibrosis and systemic sclerosis, can lead to the presence of diffuse fibrotic comets not always clearly discernible from the typical B-lines described above [10].

Finally, a focal multiple B-lines pattern can be appreciated in various other conditions such as pneumonia, pulmonary contusion, pulmonary infarction, pleural disease, and neoplasia [4].

In all such cases, to obtain a correct interpretation of the ultrasonographic data, medical history and clinical picture of the patient are mandatory.

\section{LUS in Hemodialysis}

LUS has been widely validated as an additional tool in the field of cardiology for the evaluation of cardiogenic pulmonary congestion often as a compendium to traditional echocardiographic examination [13]. 

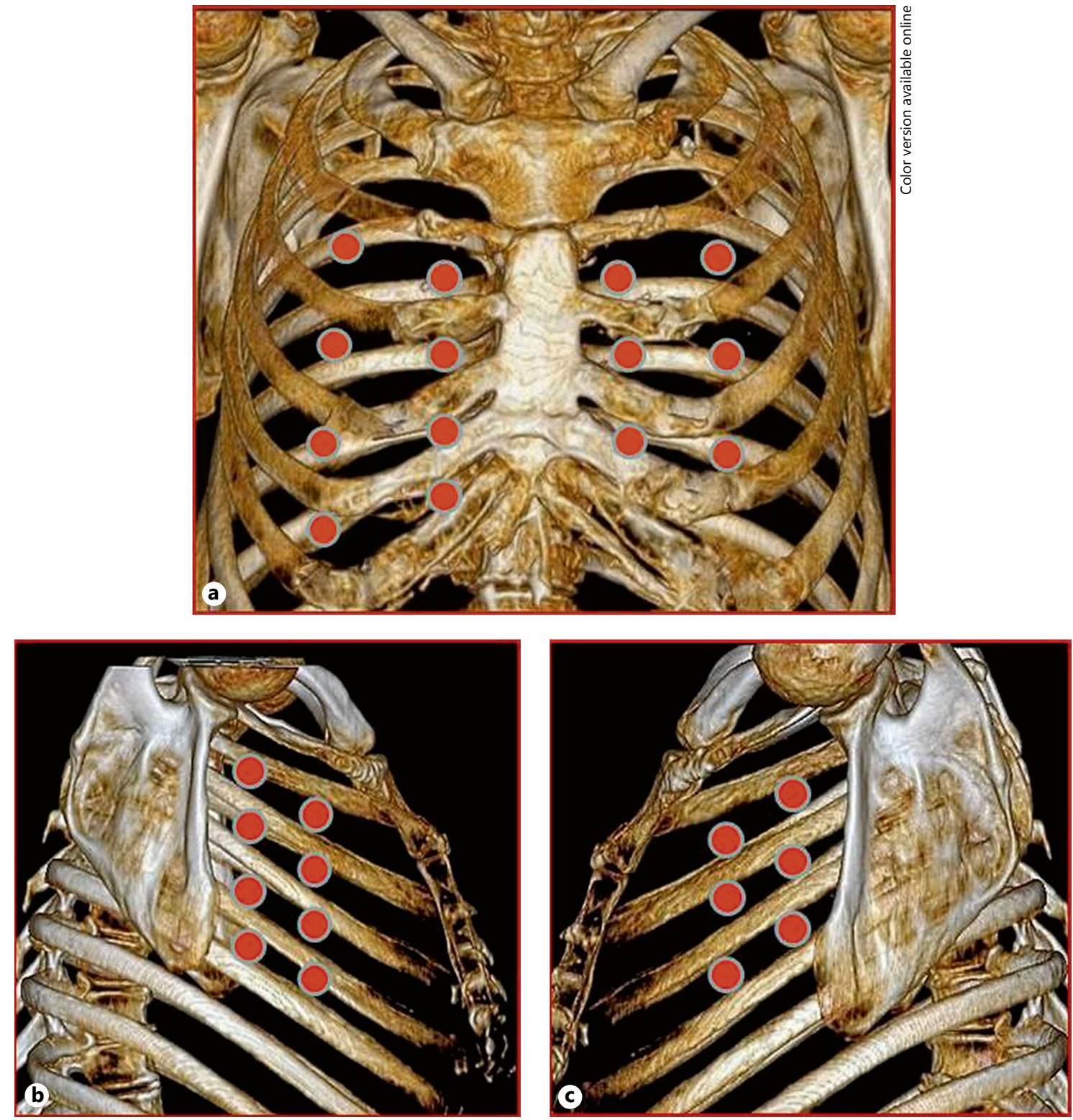

Fig. 2. CT 3D coronal imaging of the thorax: exploration points of the lung ultrasonography 28 rib interspaces technique are here indicated with red dots. a Antero-posterior view. The scanning is performed with the probe placed in the intercostal spaces along the parasternal and mid-clavicular lines in both the hemithorax

The presence of multiple B-lines is the sonographic hallmark of lung edema and a negative LUS is superior to standard chest X-ray in excluding significant pulmonary water overload [4]. The prognostic role of thoracic US in the stratification of patients with dyspnea and the presence of $\mathrm{B}$-lines at discharge have been found to be a predictor of hospital readmission and death in HF patients $[17,18]$.
(6 projections on the left, 8 on the right). b Right latero-lateral view. The transducer is placed along the anterior and mid-axillary lines from the second to the fifth intercostal space. c Left laterolateral view. The transducer is placed along the anterior and midaxillary lines from the second to the fourth intercostal space.

Using its ability to accurately estimate the EVLW, the modern LUS has extended its field of clinical application from cardiology and intensive care setting to hemodialysis where it has been proved to be useful and easy to learn $[3,19]$.

In fact, in ESRD patients, the determination and control of extracellular volume status are among the major determinants of cardiovascular outcome and the clinical 
evaluation alone has proven to be rather imprecise in estimating the correct "dry weight" [20-22].

To endorse this statement in a population of ESRD patients with heart disease, LUS has recently been confirmed to be superior in sensitivity to clinical signs of fluid overload such as lung crackles and peripheral edema: LUS revealed pulmonary congestion in $65 \%$ of the instances, while clinical signs were helpful only in $21 \%$ [23].

But what can really LUS add when compared to the other tools commonly used for the measure of fluid overload in dialysis?

LUS's great sensitivity in providing a direct measure of EVLW is well known with an analogous estimation to that of thoracic impedance but with better accuracy and precision [24].

In some ways, this peculiarity of the LUS represents both its strength and its limit, because unlike other techniques such as BIS, ultrasonographic measuring of the diameter of the IVC or on-line blood volume monitoring, it cannot directly evaluate systemic fluid volume; in other words, LUS gives an extremely focused view of pulmonary interstitial water in dialysis patients, but it is unable to provide information on the total-body volume status of the patient. This explains why, as previously reported, volemic measurement made with different techniques can be concordant in full, in part or not at all $[8,23,25]$.

Another peculiar and intriguing feature of LUS is that it has the ability to detect and estimate the degree of EVLW in a dynamic way, so that a quantitative reduction of B-lines can be appreciated in real-time during the dialysis session and in proportion to fluid volumes removed by ultrafiltration [26]. On the other side, techniques such as BIS cannot accurately evaluate water loss during hemodialysis and measuring of IVC diameter has proven to be inferior to LUS in the evaluation of the state of hydration at the end of a dialysis session [27, 28].

Furthermore, LUS can also act as a good prognostic indicator in hemodialytic patients showing a greater predictive power for hydration-related cardiovascular morbidity and mortality than classical clinical scores such as NYHA or other techniques such as BIS and echocardiography $[29,30]$.

\section{LUS and Hidden Lung Congestion}

A state of chronic volume expansion of variable degree is prevalent in patients with renal impairment and active nephrotic syndrome. In this setting, LUS has aided in the detection of asymptomatic lung edema [31].
The importance and frequency of this so-called hidden lung congestion has been recently verified in a population of ESRD patients on dialysis: in a study on 88 peritoneal dialysis patients, the thoracic US noted statistically significant mild to severe pulmonary congestion (46\%), in the absence of clinical symptoms and signs of fluid overload [32].

Again, in a population of 270 hemodialysis patients, the thoracic US revealed a moderate-to-severe pulmonary congestion in $58 \%$ of the subjects of which $38 \%$ were asymptomatic and classified as class NYHA 1; LUS findings correlated better with reduced physical activity and tolerance, regardless of NYHA classification [33].

Analogous conclusions were drawn on 51 peritoneal dialysis patients in whom asymptomatic lung congestion emerged as a crucial factor in determining the decreased physical functioning detected [34].

Accordingly, we can assume that regardless of the type of dialysis population considered (hemodialytic or peritoneal), asymptomatic pulmonary fluid overload is often present and, as it remains clinically "silent," it can over time negatively impact the quality of life and survival of ESRD patients [21].

To explain the high incidence of pulmonary congestion in dialysis patients and the role of LUS, the main pathophysiologic factors involved in the lung edema have to be considered: among them ventricular performance and pulmonary permeability are recognized as the more significant, while the systemic volume overload itself has probably a secondary role (Fig. 3). In fact, not only data from literature confirm that some degree of functional impairment of the left ventricle is very common and present in about half of the asymptomatic hemodialytic population [35]. It has also been well documented that some of the most important parameters of the LV function such as ejection fraction, left atrial volume and lung pressures are strongly related to the findings of LUS, while their associations with the total body water measured by BIS is much less significant $[8,17,19]$.

Therefore, the strong sensitivity of LUS for the early detection of pulmonary congestion in dialysis patients, especially when associated with reduced physical function, could partly derive from the pathophysiological correlation between LUS and functional disorders of the left ventricle [33].

But in order to fully understand the high frequency of hidden lung congestion in ESRD patients, the augmented lung permeability should be considered. In conditions of severe $\mathrm{CKD}$, the production of pro-inflammatory and pulmonary permeabilizing cytokines such as interleu- 
Fig. 3. Proposed physiopathologic sequence for the appearance of B-lines. Mainly heart impairment and lung-augmented permeability are responsible of EVLW expansion. The presence of different interfaces (i.e., liquid filled alveoli and normo-aerated alveoli) produces the comet tail artifacts, which can be appreciated early by chest ultrasound. With the progression of lung interstitial syndrome, the transition between pre-clinical and clinical phase occurs together with B-lines quantitative increase. EVLW, extra vascular lung water; LV, left ventricular.

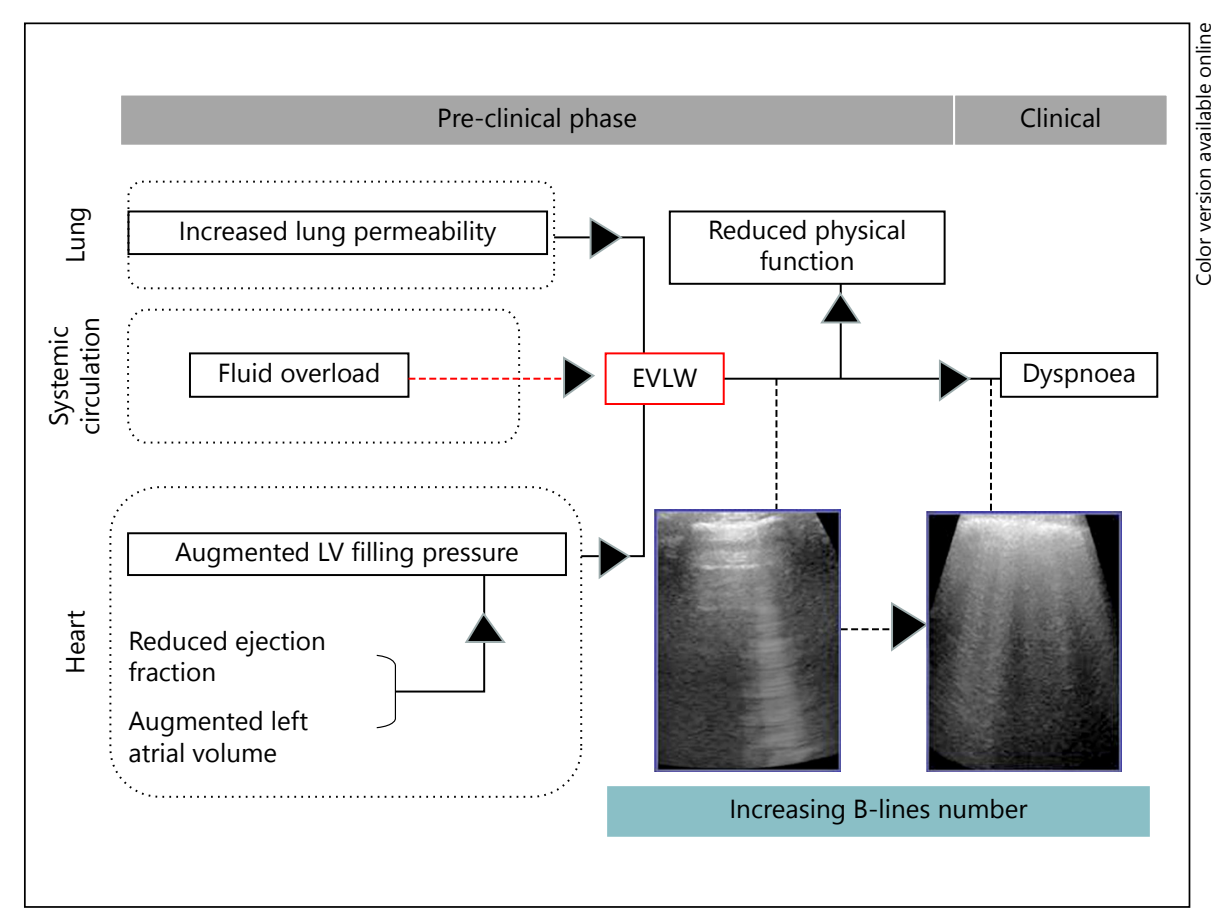

kin- 6 greatly increases. This and other mediators can then determine the expansion of the EVLW setting up the framework of the "uremic lung" and resulting in the appearance of B-lines [36, 37] (Fig. 3).

So LUS can be useful in detecting EVLW expansion both in case of increased lung permeability and underlying cardiac dysfunction, regardless of systemic volume overload or presence of symptoms. But if this diagnostic tool is widely used, what is far less clear is whether LUS can assume a pivotal role in the clinical management of the dialytic session and the real impact of the LUS in ESRD patient's therapy is still under discussion.

At present, a multicenter clinical study in hemodialysis patients with heart disease (LUST trial) is under way in order to answer this clinical question [23].

Initial results, recently published, confirm the usefulness of a therapeutic LUS-driven approach, given the low sensitivity of traditional clinical signs of fluid overload, in detecting interstitial pulmonary edema. Further evidence is necessary to explore the real impact of LUS-guided therapy on cardiovascular outcome [38].

\section{Conclusions}

Patients with ESRD on dialysis may have a significant expansion of EVLW compartment without dyspnea and/ or obvious clinical evidence of volume excess.
The reduced LV performance and increased lung permeability are determinant factors in the genesis of the hidden lung congestion.

LUS is strictly related to both these pathophysiological factors, so it is an effective tool to be used.

If correctly performed and interpreted, LUS provides high sensitivity in detecting and prognosticating ESRD patients with symptomatic and asymptomatic lung edema. But sensitivity and specificity of LUS findings of lung edema remain in need of further discussion and definition, especially in differentiating those patients with other conditions like pneumonia, infarction, pleural disease and bronchogenic neoplasia.

\section{Disclosure Statement}

The authors have declared that no conflict of interest exists.

\section{Compliance with Ethical Standards}

Human and animal rights: this article does not contain any studies with human participants or animals performed by any of the authors.

Informed consent: informed consent was obtained from all individual participants included in the article.

All authors have read and approved the manuscript. 


\section{References}

1 Hecking M, Rayner H, Wabel P: What are the consequences of volume expansion in chronic dialysis patients?: defining and measuring fluid overload in hemodialysis patients. Semin Dial 2015;28:242-247.

2 Ronco C, Kaushik M, Valle R, Aspromonte N, Peacock WF 4th: Diagnosis and management of fluid overload in heart failure and cardiorenal syndrome: the " $5 \mathrm{~B}$ " approach. Semin Nephrol 2012;32:129-141.

3 Ross DW, Barnett RL, Shah HH: Lung ultrasonography: a novel clinical tool to consider in nephrology. Kidney Int 2016;89: 720-721.

4 Volpicelli G, Elbarbary M, Blaivas M, et al; International Liaison Committee on Lung Ultrasound (ILC-LUS) for International Consensus Conference on Lung Ultrasound (ICC-LUS): International evidence-based recommendations for point-of-care lung ultrasound. Intensive Care Med 2012;38:577-591.

5 Ziskin MC, Thickman DI, Goldenberg NJ, Lapayowker MS, Becker JM: The comet tail artifact. J Ultrasound Med 1982;1:1-7.

6 Lichtenstein D, Mézière G, Biderman P, Gepner A, Barré O: The comet-tail artifact. An ultrasound sign of alveolar-interstitial syndrome. Am J Respir Crit Care Med 1997;156: 1640-1646.

7 Picano E, Frassi F, Agricola E, Gligorova S, Gargani L, Mottola G: Ultrasound lung comets: a clinically useful sign of extravascular lung water. J Am Soc Echocardiogr 2006;19: 356-363.

8 Mallamaci F, Benedetto FA, Tripepi R, et al: Detection of pulmonary congestion by chest ultrasound in dialysis patients. JACC Cardiovasc Imaging 2010;3:586-594.

9 Soldati G, Copetti R, Sher S: Can lung comets be counted as "objects"? JACC Cardiovasc Imaging 2011;4:438-439.

10 Picano E, Pellikka PA: Ultrasound of extravascular lung water: a new standard for pulmonary congestion. Eur Heart J 2016;37: 2097-2104.

11 Spinelli A, Vinci B, Tirella A, et al: Realization of a poro-elastic ultrasound replica of pulmonary tissue. Biomatter 2012;2:37-42.

12 Volpicelli G, Mussa A, Garofalo G, et al: Bedside lung ultrasound in the assessment of alveolar-interstitial syndrome. Am J Emerg Med 2006;24:689-696.

13 Jambrik Z, Monti S, Coppola V, et al: Usefulness of ultrasound lung comets as a nonradio- logic sign of extravascular lung water. Am J Cardiol 2004;93:1265-1270.

14 Gargani L, Volpicelli G: How I do it: lung ultrasound. Cardiovasc Ultrasound 2014;12:25.

15 Weitzel WF, Hamilton J, Wang X, et al: Quantitative lung ultrasound comet measurement: method and initial clinical results. Blood $\mathrm{Pu}$ rif 2015;39:37-44.

16 Lichtenstein DA: Lung ultrasound in the critically ill. Ann Intensive Care 2014;4:1.

17 Frassi F, Gargani L, Tesorio P, Raciti M, Mottola G, Picano E: Prognostic value of extravascular lung water assessed with ultrasound lung comets by chest sonography in patients with dyspnea and/or chest pain. J Card Fail 2007;13:830-835

18 Cogliati C, Casazza G, Ceriani E, et al: Lung ultrasound and short-term prognosis in heart failure patients. Int J Cardiol 2016;218:104108.

19 Enghard P, Rademacher S, Nee J, et al: Simplified lung ultrasound protocol shows excellent prediction of extravascular lung water in ventilated intensive care patients. Crit Care 2015; 19:36.

20 Ozkahya M, Ok E, Toz H, et al: Long-term survival rates in haemodialysis patients treated with strict volume control. Nephrol Dial Transplant 2006;21:3506-3513.

21 Kalantar-Zadeh K, Regidor DL, Kovesdy CP, et al: Fluid retention is associated with cardiovascular mortality in patients undergoing long-term hemodialysis. Circulation 2009; 119:671-679.

22 Palmer BF, Henrich WL: Recent advances in the prevention and management of intradialytic hypotension. J Am Soc Nephrol 2008;19: 8-11.

23 Torino C, Gargani L, Sicari R, et al: The agreement between auscultation and lung ultrasound in hemodialysis patients: the LUST study. Clin J Am Soc Nephrol 2016;11:20052011.

24 Maines M, Catanzariti D, Angheben C, et al: Intrathoracic impedance and ultrasound lung comets in heart failure deterioration monitoring. Pacing Clin Electrophysiol 2011;34:968974.

25 Zoccali C, Tripepi R, Torino C, Bellantoni M, Tripepi G, Mallamaci F: Lung congestion as a risk factor in end-stage renal disease. Blood Purif 2013;36:184-191.

26 Noble VE, Murray AF, Capp R, Sylvia-Reardon MH, Steele DJ, Liteplo A: Ultrasound as- sessment for extravascular lung water in patients undergoing hemodialysis. Time course for resolution. Chest 2009;135:1433-1439.

27 Di Iorio BR, Scalfi L, Terracciano V, Bellizzi $\mathrm{V}$ : A systematic evaluation of bioelectrical impedance measurement after hemodialysis session. Kidney Int 2004;65:2435-2440.

28 Basso F, Milan Manani S, Cruz DN, et al: Comparison and reproducibility of techniques for fluid status assessment in chronic hemodialysis patients. Cardiorenal Med 2013;3:104-112.

29 Siriopol D, Hogas S, Voroneanu L, et al: Predicting mortality in haemodialysis patients: a comparison between lung ultrasonography, bioimpedance data and echocardiography parameters. Nephrol Dial Transplant 2013; 28:2851-2859.

30 Zoccali C, Torino C, Tripepi R, et al: Pulmonary congestion predicts cardiac events and mortality in ESRD. J Am Soc Nephrol 2013; 24:639-646.

31 Marino F, Martorano C, Tripepi R, et al: Subclinical pulmonary congestion is prevalent in nephrotic syndrome. Kidney Int 2016;89: 421-428.

32 Panuccio V, Enia G, Tripepi R, et al: Chest ultrasound and hidden lung congestion in peritoneal dialysis patients. Nephrol Dial Transplant 2012;27:3601-3605.

33 Enia G, Torino C, Panuccio V, et al: Asymptomatic pulmonary congestion and physical functioning in hemodialysis patients. Clin J Am Soc Nephrol 2013;8:1343-1348.

34 Enia G, Tripepi R, Panuccio V, et al: Pulmonary congestion and physical functioning in peritoneal dialysis patients. Perit Dial Int 2012;32:531-536.

35 Zoccali C, Benedetto FA, Mallamaci F, et al: Prognostic value of echocardiographic indicators of left ventricular systolic function in asymptomatic dialysis patients. J Am Soc Nephrol 2004;15:1029-1037.

36 Hasuike Y, Nonoguchi H, Ito K, et al: Interleukin-6 is a predictor of mortality in stable hemodialysis patients. Am J Nephrol 2009;30: 389-398

37 Scheel PJ, Liu M, Rabb H: Uremic lung: new insights into a forgotten condition. Kidney Int 2008;74:849-851.

38 Sherman RA: Crackles and comets: lung ultrasound to detect pulmonary congestion in patients on dialysis is coming of age. Clin J Am Soc Nephrol 2016;11:1924-1926. 